\title{
Properties of Sakaguchi Kind Functions Associated with Bessel Function
}

\author{
H. Priya*, B. Srutha Keerthi \\ Department of Mathematics, School of Advanced Sciences, VIT Chennai Campus, India
}

Received August 22, 2021; Revised October 19, 2021; Accepted November 11, 2021

Cite This Paper in the following Citation Styles

(a): [1] H.Priya, B. Srutha Keerthi, "Properties of Sakaguchi Kind Functions Associated with Bessel Function," Mathematics and Statistics, Vol.9, No.6, pp. 1011-1018, 2021. DOI: 10.13189/ms.2021.090617

(b): H.Priya, B. Srutha Keerthi, (2021). Properties of Sakaguchi Kind Functions Associated with Bessel Function. Mathematics and Statistics, 9(6), 10111018. DOI: $10.13189 / \mathrm{ms} .2021 .090617$

Copyright $\odot 2021$ by authors, all rights reserved. Authors agree that this article remains permanently open access under the terms of the Creative Commons Attribution License 4.0 International License

Abstract The aim of the paper is to obtain the First Hankel Determinant and the Second Hankel determinant. We shall make use of few lemmas which are based on Caratheodory's class of analytic functions. We establish a new Sakaguchi class

$$
\frac{(1-t)\left[\rho \xi^{2}\left(N_{\nu, q}^{\lambda} f(\xi)\right)^{\prime \prime}+\xi\left(N_{\nu, q}^{\lambda} f(\xi)\right)^{\prime}\right]}{\rho \xi\left[\left(N_{\nu, q}^{\lambda} f(\xi)\right)^{\prime}-t\left(N_{\nu, q}^{\lambda} f(t \xi)\right)^{\prime}\right]+(1-\rho)\left[N_{\nu, q}^{\lambda} f(\xi)-N_{\nu, q}^{\lambda} f(t \xi)\right]} \prec \Psi(\xi)
$$

of univalent function, further we estimate the sharp bound for initial coefficients $a_{2}$ and $a_{3}$ using the Bessel function expansion. We have discussed about the coefficient $a_{4}$ as well for the Second Hankel Determinant. The results are obtained for Sakaguchi kind. Our results travel along exploring the stages of Hankel Determinants. Various types of technologies like wire, optical or other electromagnetic systems are used for the transmission of data in one device to another. Filters play an important role in the process that can remove disorted signals. By using different parameter values for the function belongs to Sakaguchi kind of functions the Low pass filter and High pass filter can be designed and that can be done by the coefficient estimates.

Keywords Coefficient Inequality, Subordination Techniques, Two Kinds of Bessel Function, Second Hankel Determinant AMS Subject Classification rimary: 30C45, 30C80

\section{Introduction}

Let $\mathcal{A}$ denote the class of analytic functions of the form:

$$
f(\xi)=\xi+\sum_{\kappa=2}^{\infty} a_{\kappa} \xi^{\kappa}, \quad \xi \in \mathbb{U}=\{\xi \in \mathbb{C}:|\xi|<1\}
$$

and $\mathcal{S}$ be the subclass of $\mathcal{A}$ which are univalent in $\mathbb{U}$. If $\kappa \in \mathcal{A}$ is represented as:

$$
\kappa(\xi)=\xi+\sum_{\kappa=2}^{\infty} b_{\kappa} \xi^{\kappa}, \quad \xi \in \mathbb{U}
$$


then, the Hadamard product of $f$ and $\kappa$ is constructed as follows:

$$
(f \times \kappa)(\xi)=\xi+\sum_{\kappa=2}^{\infty} a_{\kappa} b_{\kappa} \xi^{\kappa}, \quad \xi \in \mathbb{U}
$$

If the function $F$ is univalent in $\mathbb{U}$, then the following holds (see [1] and [2]):

$$
f(\xi) \prec F(\xi) \Leftrightarrow f(0)=F(0) \text { and } f(\mathbb{U}) \subset F(\mathbb{U}) .
$$

The infinite series is given by:

$$
J_{\nu}(\xi)=\sum_{\kappa \geq 0} \frac{(-1)^{\kappa}\left(\frac{\xi}{2}\right)^{2 \kappa+\nu}}{\kappa ! \Gamma(\kappa+\nu+1)}, \quad \xi \in \mathbb{C},(\nu \in \mathbb{R}),
$$

where $\Gamma$ denotes the Gamma function.[3] the normalized Bessel function of the first kind $g_{\nu}: \mathbb{U} \rightarrow \mathbb{C}$ defined by (see also [4] - [6])

$$
\begin{aligned}
g_{\nu}(\xi) & =2^{\nu} \Gamma(\nu+1) \xi^{1-\frac{\nu}{2}} J_{\nu}\left(\xi^{\frac{1}{2}}\right) \\
& =\xi+\sum_{\kappa=2}^{\infty} \frac{(-1)^{\kappa-1} \Gamma(\nu+1)}{4^{\kappa-1}(\kappa-1) ! \Gamma(\kappa+\nu)} \xi^{\kappa}, \quad \xi \in \mathbb{U},(\nu \in \mathbb{R})
\end{aligned}
$$

For the strict inquality $0<q<1, g_{\nu}$ is defined by;

$$
\begin{aligned}
\partial_{q} g_{\nu}(\xi) & =\partial_{q}\left\{\xi+\sum_{\kappa=2}^{\infty} \frac{(-1)^{\kappa-1} \Gamma(\nu+1)}{4^{\kappa-1}(\kappa-1) ! \Gamma(\kappa+\nu)} \xi^{\kappa}\right\} \\
& =\frac{g_{\nu}(q \xi)-g_{\nu}(\xi)}{\xi(q-1)} \\
& =1+\sum_{\kappa=2}^{\infty} \frac{(-1)^{\kappa-1} \Gamma(\nu+1)}{4^{\kappa-1}(\kappa-1) ! \Gamma(\kappa+\nu)}[\kappa, q] \xi^{\kappa-1}, \quad \xi \in \mathbb{U}
\end{aligned}
$$

where

$$
[\kappa, q]=\frac{1-q^{\kappa}}{1-q}=1+\sum_{j=1}^{\kappa-1} q^{j},[0, q]=0
$$

using (4), the next two products are obtained:

(i) The q-shifted fractional for a positive integer $k$ is given by;

$$
[\kappa, q] != \begin{cases}1, & \text { if } \kappa=0 \\ {[1, q][2, q][3, q] \ldots[\kappa, q],} & \text { if } \kappa \in \mathbb{N}\end{cases}
$$

(ii) The q-generalised Pochhammer symbol for a positive number $r$ is defined by;

$$
[r, q]_{\kappa}= \begin{cases}1, & \text { if } \kappa=0 \\ {[r, q][r+1, q] \ldots[r+\kappa-1, q],} & \text { if } \kappa \in \mathbb{N}\end{cases}
$$

For the conditions $\nu>0, \lambda>-1$, and $0<q<1$, we can define the function $I_{\nu, q}^{\lambda}: \mathbb{U} \rightarrow \mathbb{C}$ by;

$$
I_{\nu, q}^{\lambda}(\xi)=\xi+\sum_{\kappa=2}^{\infty} \frac{(-1)^{\kappa-1} \Gamma(\nu+1)}{4^{\kappa-1}(\kappa-1) ! \Gamma(\kappa+\nu)} \frac{[\kappa, q] !}{[\lambda+1, q]_{\kappa-1}} \xi^{\kappa}, \quad \xi \in \mathbb{U} .
$$

The Hankel determinants $H_{\xi}(1)=a_{3}-a_{2}^{2}$ and $H_{\xi}(2)=a_{2} a_{4}-a_{3}^{2}$ are discussed.

Remark 1. A simple reckoning shows that:

$I_{\nu, q}^{\lambda}(\xi) \times M_{q \lambda+1}(\xi)=\xi \partial_{q} g_{\nu} \quad \xi \in \mathbb{U}$, where $M_{q \lambda+1}$ is given by:

$$
M_{q \lambda+1}(\xi)=\xi+\sum_{\kappa=2}^{\infty} \frac{[\lambda+1, q]_{\kappa-1}}{[\kappa-1, q] !} \xi^{\kappa} \xi \in \mathbb{U}
$$


By making using of idealogy of q-derivative, we instigate the linear operator $N_{\nu, q}^{\lambda}: \mathcal{A} \rightarrow \mathcal{A}$ defined by:

$$
N_{\nu, q}^{\lambda} f(\xi)=I_{\nu, q}^{\lambda}(\xi) \times f(\xi)=\xi+\sum_{\kappa=2}^{\infty} \Psi_{\kappa} a_{\kappa} \xi^{\kappa}, \quad \xi \in \mathbb{U}
$$

with the conditions $(\nu>0, \lambda>-1,0<q<1)$,

where

$$
\Psi_{\kappa}=\frac{(-1)^{\kappa-1} \Gamma(\nu+1)}{4^{\kappa-1}(\kappa-1) ! \Gamma(\kappa+\nu)} \frac{[\kappa, q] !}{[\lambda+1, q]_{\kappa-1}}
$$

Remark 2. From (5),

(i) $[\lambda+1, q] N_{\nu, q}^{\lambda} f(\xi)=[\lambda, q] N_{\nu, q}^{\lambda+1} f(\xi)+q^{\lambda} \xi \partial_{q}\left(N_{\nu, q}^{\lambda+1} f(\xi)\right), \quad \xi \in \mathbb{U}$

(ii) $\lim _{q \rightarrow 1^{-}} N_{\nu, q}^{\lambda} f(\xi)=I_{\nu, 1}^{\lambda} \times f(\xi)=\xi+\sum_{\kappa=2}^{\infty} \frac{\kappa !}{[\lambda+1]_{\kappa-1}} \frac{(-1)^{\kappa-1} \Gamma(\nu+1)}{4^{\kappa-1}(\kappa-1) ! \Gamma(\kappa+\nu)} a_{\kappa} \xi^{\kappa}$,

$\xi \in \mathbb{U}$

Proof. (i)To prove we consider RHS and arrive at the LHS

$$
\begin{aligned}
& {[\lambda, q] N_{\nu, q}^{\lambda+1} f(\xi)+q^{\lambda} \xi \partial_{q}\left(N_{\nu, q}^{\lambda+1} f(\xi)\right)=[\lambda, q]\left[\xi+\sum_{\kappa=2}^{\infty} \Upsilon \frac{[\kappa, q] !}{[\lambda+2, q]_{\kappa-1}} a_{\kappa} \xi^{\kappa}\right]} \\
& +q^{\lambda}\left[\xi+\sum_{\kappa=2}^{\infty} \Upsilon \frac{[\kappa, q] ![\kappa, q]}{[\lambda+2, q]_{\kappa-1}} a_{\kappa} \xi^{\kappa}\right] \\
& =\quad \xi\left\{[\lambda, q]+q^{\lambda}\right\}+\sum_{\kappa=2}^{\infty}\left\{\Upsilon \frac{[\kappa, q] !}{[\lambda+2, q]_{\kappa-1}} a_{\kappa} \xi^{\kappa}\right\}\left\{[\lambda, q]+q^{\lambda}[\kappa, q]\right\} \\
& =\xi\left\{\frac{1-q^{\lambda}}{1-q}+q^{\lambda}\right\}+\frac{(-1)^{2-1} \Gamma(\nu+1)}{4^{2-1}(2-1) ! \Gamma(2+\nu)} \frac{[2, q] !}{[\lambda+2, q]_{2-1}} a_{2} \xi^{2}\left\{[\lambda, q]+q^{\lambda}[2, q]\right\} \\
& +\frac{(-1)^{3-1} \Gamma(\nu+1)}{4^{3-1}(3-1) ! \Gamma(3+\nu)} \frac{[3, q] !}{[\lambda+2, q]_{3-1}} a_{3} \xi^{3}\left\{[\lambda, q]+q^{\lambda}[3, q]\right\}+\cdots \\
& =[\lambda+1, q] \xi+\sum_{\kappa=2}^{\infty} \Upsilon \frac{[\kappa, q] ![\lambda+1, q]}{[\lambda+1, q]_{\kappa-1}} a_{\kappa} \xi^{\kappa} \\
& =[\lambda+1, q]\left[\xi+\sum_{\kappa=2}^{\infty} \Upsilon \frac{[\kappa, q] !}{[\lambda+1, q]_{\kappa-1}} a_{\kappa} \xi^{\kappa}\right] \\
& =[\lambda+1, q] N_{\nu, q}^{\lambda} f(\xi)
\end{aligned}
$$

(ii)W.K.T,

$$
N_{\nu, q}^{\lambda} f(\xi)=\xi+\sum_{\kappa=2}^{\infty} \Upsilon \frac{[\kappa, q] !}{[\lambda+1, q]_{\kappa-1}} a_{\kappa} \xi^{\kappa}
$$

Applying limit, we get

$$
\begin{gathered}
\lim _{q \rightarrow 1^{-}} N_{\nu, q}^{\lambda} f(\xi)=\xi+\sum_{\kappa=2}^{\infty} \Upsilon a_{\kappa} \xi^{\kappa} \frac{(1,1)(2,1)(3,1)(4,1) \ldots(\kappa, 1)}{[\lambda+1,1][\lambda+2,1] \ldots[\lambda+(\kappa-1), 1]} \\
=\xi+\sum_{\kappa=2}^{\infty} \Upsilon \frac{\kappa !}{[\lambda+1]_{\kappa-1}} a_{\kappa} \xi^{\kappa}
\end{gathered}
$$

where $\Upsilon=\frac{(-1)^{\kappa-1} \Gamma(\nu+1)}{4^{\kappa-1}(\kappa-1) ! \Gamma(\kappa+\nu)}$ 
Now, we bring in the class of functions $M_{\nu, q}^{\lambda}(\rho, t, \Psi)$ as follows

Definition 3. Let $\Psi(\xi)=1+B_{1} \xi+B_{2} \xi^{2}+\ldots, \quad \xi \in \mathbb{U}$, with the condition $B_{1}>0$, then the function $f \in \mathcal{A}$ is said to be in the class $M_{\nu, q}^{\lambda}(\rho, t, \Psi)$ if the function

$$
\frac{(1-t)\left[\rho \xi^{2}\left(N_{\nu, q}^{\lambda} f(\xi)\right)^{\prime \prime}+\xi\left(N_{\nu, q}^{\lambda} f(\xi)\right)^{\prime}\right]}{\rho \xi\left[\left(N_{\nu, q}^{\lambda} f(\xi)\right)^{\prime}-t\left(N_{\nu, q}^{\lambda} f(t \xi)\right)^{\prime}\right]+(1-\rho)\left[N_{\nu, q}^{\lambda} f(\xi)-N_{\nu, q}^{\lambda} f(t \xi)\right]}
$$

is analytic in $\mathbb{U}$ and satisfies:

$$
\frac{(1-t)\left[\rho \xi^{2}\left(N_{\nu, q}^{\lambda} f(\xi)\right)^{\prime \prime}+\xi\left(N_{\nu, q}^{\lambda} f(\xi)\right)^{\prime}\right]}{\rho \xi\left[\left(N_{\nu, q}^{\lambda} f(\xi)\right)^{\prime}-t\left(N_{\nu, q}^{\lambda} f(t \xi)\right)^{\prime}\right]+(1-\rho)\left[N_{\nu, q}^{\lambda} f(\xi)-N_{\nu, q}^{\lambda} f(t \xi)\right]} \prec \Psi(\xi)
$$

$(\nu>0, \lambda>-1,0<q<1,0 \leq \rho \leq 1,|t| \leq 1$ but $t \neq 1)$

In this paper, we obtain the Fekete-Szego inequalities and Second Hankel Determinant for the function of the class $M_{\nu, q}^{\lambda}(\rho, t, \Psi)$.

\section{First Hankel Dterminant Problem}

Lemma 4. ([8], Lemma 3) If $p(\xi)=1+C_{1} \xi+C_{2} \xi^{2}+\ldots \in \mathcal{P}$ and $\alpha$ is said to be a complex number, then

$$
\max \left|C_{2}-\alpha C_{1}^{2}\right|=2 \max \{1 ;|2 \alpha-1|\} .
$$

Lemma 5. ([9], Lemma1) If $p(\xi)=1+C_{1} \xi+C_{2} \xi^{2}+\ldots \in \in \mathcal{P}$, then

$$
\left|C_{2}-\alpha C_{1}^{2}\right| \leq \begin{cases}-4 \alpha+2, & \text { if } \alpha \leq 0 \\ 2, & \text { if } 0 \leq \alpha \leq 1 \\ 4 \alpha-2, & \text { if } \alpha \geq 1\end{cases}
$$

When $\alpha<0$ or $\alpha>1$, the equality holds iff

$$
p(\xi)=\frac{1+\xi}{1-\xi}
$$

or one of its rotations.

If the strict inequality $0<\alpha<1$ is considered, then the equality holds if and only if

$$
p(\xi)=\frac{1+\xi^{2}}{1-\xi^{2}}
$$

or one of its rotations.

If $\alpha=0$, the equality holds if and only if:

$$
p(\xi)=\left(\frac{1}{2}+\frac{\lambda}{2}\right) \frac{1+\xi}{1-\xi}+\left(\frac{1}{2}-\frac{\lambda}{2}\right) \frac{1-\xi}{1+\xi},
$$

with $0 \leq \lambda \leq 1$, or one of its rotations.

If $\alpha=1$, the equality holds iff:

$$
\frac{1}{p(\xi)}=\left(\frac{1}{2}+\frac{\lambda}{2}\right) \frac{1+\xi}{1-\xi}+\left(\frac{1}{2}-\frac{\lambda}{2}\right) \frac{1-\xi}{1+\xi},
$$

with $0 \leq \lambda \leq 1$. From the reference $([9]$, pages $162-163)$, it can be improved in the following way when $0<\alpha<1$ :

$$
\left|C_{2}-\alpha C_{1}^{2}\right|+\alpha\left|C_{1}^{2}\right| \leq 2, \text { if } 0<\alpha \leq \frac{1}{2}
$$

and

$$
\left|C_{2}-\alpha C_{1}^{2}\right|+(1+\alpha)\left|C_{1}^{2}\right| \leq 2, \text { if } \frac{1}{2} \leq \alpha<1
$$

Theorem 6. When $f(\xi)$ given by (1) belongs to the class $M_{\nu, q}^{\lambda}(\rho, t, \Psi)$, with $\Psi(\xi)$ which satisfies Definition (1), and $\mu$ is said to be a complex number, then:

$$
\left|a_{3}-\mu a_{2}^{2}\right| \leq \frac{B_{1}}{\Psi_{3}(1+2 \rho)\left(3-u_{3}\right)} \max \left\{1 ;\left|\frac{B_{2}}{B_{1}}+\frac{B_{1} u_{2}}{\left(2-u_{2}\right)}-\frac{\mu B_{1} \Psi_{3}\left(3-u_{3}\right)(1+2 \rho)}{\Psi_{2}^{2}(1+\rho)^{2}\left(2-u_{2}\right)^{2}}\right|\right\}
$$

where $\Psi_{\kappa}, \kappa \in\{2,3\}$, are given by (6). 
Proof. When $f(\xi) \in M_{\nu, q}^{\lambda}(\rho, t, \Psi)$, then we observe that there exists a Schwarz function $W$, which is analytic in $\mathbb{U}$, with $W(0)=0$ and $|W(\xi)|<1$, $z \in \mathbb{D}$, such that:

$$
\frac{(1-t)\left[\rho \xi^{2}\left(N_{\nu, q}^{\lambda} f(\xi)\right)^{\prime \prime}+\xi\left(N_{\nu, q}^{\lambda} f(\xi)\right)^{\prime}\right]}{\rho \xi\left[\left(N_{\nu, q}^{\lambda} f(\xi)\right)^{\prime}-t\left(N_{\nu, q}^{\lambda} f(t \xi)\right)^{\prime}\right]+(1-\rho)\left[N_{\nu, q}^{\lambda} f(\xi)-N_{\nu, q}^{\lambda} f(t \xi)\right]}=\Psi(W(\xi)), \quad \xi \in \mathbb{U}
$$

Since $W$ is a Schwarz function, it follows that the function $p_{1}$ defined by;

$$
p_{1}(\xi)=\frac{1+W(\xi)}{1-W(\xi)}=1+C_{1} \xi+C_{2} \xi^{2}+\ldots, \quad \xi \in \mathbb{U}
$$

belongs to $\mathcal{P}$. By defining the function $p$ by:

$$
\begin{aligned}
p(\xi) & =\frac{(1-t)\left[\rho \xi^{2}\left(N_{\nu, q}^{\lambda} f(\xi)\right)^{\prime \prime}+\xi\left(N_{\nu, q}^{\lambda} f(\xi)\right)^{\prime}\right]}{\rho \xi\left[\left(N_{\nu, q}^{\lambda} f(\xi)\right)^{\prime}-t\left(N_{\nu, q}^{\lambda} f(t \xi)\right)^{\prime}\right]+(1-\rho)\left[N_{\nu, q}^{\lambda} f(\xi)-N_{\nu, q}^{\lambda} f(t \xi)\right]} \\
& =1+d_{1} \xi+d_{2} \xi^{2}+\ldots
\end{aligned}
$$

In view of (9) and (10), we have:

$$
p(\xi)=\Psi\left(\frac{p_{1}(\xi)-1}{p_{1}(\xi)+1}\right), \xi \in \mathbb{U}
$$

By making use of (10), we easily obtain:

$$
\frac{p_{1}(\xi)-1}{p_{1}(\xi)+1}=\frac{1}{2}\left[C_{1} \xi+\left(C_{1}-\frac{C_{1}^{2}}{2}\right) \xi^{2}+\left(C_{3}+\frac{C_{1}^{3}}{4}-C_{1} C_{2}\right) \xi^{3}+\ldots\right], \xi \in \mathbb{U}
$$

therefore

$$
\Psi\left(\frac{p_{1}(\xi)-1}{p_{1}(\xi)+1}\right)=1+\frac{1}{2} B_{1} C_{1} \xi+\left(\frac{1}{2} B_{1}\left(C_{2}-\frac{C_{1}^{2}}{2}\right)+\frac{1}{4} B_{2} C_{1}^{2}\right) \xi^{2}+\ldots, \xi \in \mathbb{U}
$$

and from (12), we obtain

$$
\begin{gathered}
d_{1}=\frac{1}{2} B_{1} C_{1} \\
d_{2}=\frac{1}{2} B_{1}\left(C_{2}-\frac{C_{1}^{2}}{2}\right)+\frac{1}{4} B_{2} C_{1}^{2} \\
d_{3}=\frac{1}{2} B_{1}\left(C_{3}+\frac{C_{1}^{3}}{4}-C_{1} C_{2}\right)+\frac{1}{2} B_{2} C_{1}\left(C_{2}-\frac{C_{1}^{2}}{2}\right)
\end{gathered}
$$

On the other hand, from (11), according to (5), it follows that

$$
\begin{gathered}
d_{1}=(1+\rho)\left(2-u_{2}\right) \Psi_{2} a_{2} \\
d_{2}=(1+2 \rho) \Psi_{3} a_{3}\left(3-u_{3}\right)-(1+\rho)^{2} \Psi_{2}^{2} a_{2}^{2}\left(2-u_{2}\right) u_{2} \\
d_{3}=(1+3 \rho) \Psi_{4} a_{4}\left(4-u_{4}\right)-\Psi_{2} a_{2} \Psi_{3} a_{3}\left[(1+\rho)\left(2-u_{2}\right) u_{3}+(1+\rho)(1+2 \rho)\left(3-u_{3}\right) u_{2}\right]+(1+\rho)^{3} \Psi_{2}^{3} a_{2}^{3} u_{2}^{2}\left(2-u_{2}\right)
\end{gathered}
$$

and combning $d_{1}, d_{2}$ and $d_{3}$ values, we have

$$
\begin{gathered}
a_{2}=\frac{B_{1} C_{1}}{2(1+\rho)\left(2-u_{2}\right) \Psi_{2}} \\
a_{3}=\frac{B_{1}}{2(1+2 \rho)\left(3-u_{3}\right) \Psi_{3}}\left[C_{2}-\frac{C_{1}^{2}}{2}+\frac{1}{2} \frac{B_{2}}{B_{1}} C_{1}^{2}+\frac{B_{1} C_{1}^{2} u_{2}}{2\left(2-u_{2}\right)}\right] \\
a_{4}=\frac{1}{2(1+3 \rho)\left(4-u_{4}\right) \Psi_{4}}\left[B_{1}\left(C_{3}+\frac{C_{1}^{3}}{4}-C_{1} C_{2}\right)+B_{2} C_{1}\left(C_{2}-\frac{C_{1}^{2}}{2}\right)\right. \\
+\left(\left(2-u_{2}\right) u_{3}+(1+2 \rho)\left(3-u_{3}\right) u_{2}\right)\left(\frac{B_{1}^{2} C_{1}}{2(1+2 \rho)\left(3-u_{3}\right)\left(2-u_{2}\right)}\right) \\
\left.\left(C_{2}-\frac{C_{1}^{2}}{2}+\frac{B_{2} C_{1}^{2}}{2 B_{1}}+\frac{B_{1} C_{1}^{2} u_{2}}{2\left(2-u_{2}\right)}\right)-\frac{B_{1}^{3} C_{1}^{3} u_{2}^{2}}{4\left(2-u_{2}\right)^{2}}\right]
\end{gathered}
$$

Therefore,

$$
a_{3}-\mu a_{2}^{2}=\frac{B_{1}}{2 \Psi_{3}(1+2 \rho)\left(3-u_{3}\right)}\left\{C_{2}-\alpha C_{1}^{2}\right\}
$$

where

$$
\alpha=\frac{1}{2}\left[1-\frac{B_{2}}{B_{1}}-\frac{B_{1} u_{2}}{\left(2-u_{2}\right)}+\frac{\mu B_{1} \Psi_{3}\left(3-u_{3}\right)(1+2 \rho)}{\Psi_{2}^{2}(1+\rho)^{2}\left(2-u_{2}\right)^{2}}\right]
$$

and by making use of the Lemma 1, the upcomming results are discussed. 
Theorem 7. If $f(\xi)$ given by (1), with $\Psi(\xi)$ satisfyies Definition (1) and $\mu, B_{2} \in \mathbb{R}$ and $0 \leq \rho \leq 1$ and $|t| \leq 1$ but $t \neq 1$, belongs to the class $M_{\nu, q}^{\lambda}(\rho, t, \Psi)$ then

$$
\left|a_{3}-\mu a_{2}^{2}\right| \leq \begin{cases}\frac{B_{1}}{\Psi_{3}(1+2 \rho)\left(3-u_{3}\right)}\left[\frac{B_{2}}{B_{1}}+\frac{B_{1} u_{2}}{\left(2-u_{2}\right)}-\frac{\mu B_{1} \Psi_{3}\left(3-u_{3}\right)(1+2 \rho)}{\Psi_{2}^{2}(1+\rho)^{2}\left(2-u_{2}\right)^{2}}\right], & \text { if } \mu \leq \sigma_{1} \\ \frac{B_{1}}{\Psi_{3}(1+2 \rho)\left(3-u_{3}\right)}, & \text { if } \sigma_{1} \leq \mu \leq \sigma_{2} \\ \frac{-B_{1}}{\Psi_{3}(1+2 \rho)\left(3-u_{3}\right)}\left[\frac{B_{2}}{B_{1}}+\frac{B_{1} u_{2}}{\left(2-u_{2}\right)}-\frac{\mu B_{1} \Psi_{3}\left(3-u_{3}\right)(1+2 \rho)}{\Psi_{2}^{2}(1+\rho)^{2}\left(2-u_{2}\right)^{2}}\right], & \text { if } \mu \geq \sigma_{2} .\end{cases}
$$

with

$$
\begin{aligned}
& \sigma_{1}=\frac{\Psi_{2}^{2}(1+\rho)^{2}\left(2-u_{2}\right)^{2}}{B_{1} \Psi_{3}\left(3-u_{3}\right)(1+2 \rho)}\left[\frac{B_{2}}{B_{1}}+\frac{B_{1} u_{2}}{\left(2-u_{2}\right)}-1\right] \\
& \sigma_{2}=\frac{\Psi_{2}^{2}(1+\rho)^{2}\left(2-u_{2}\right)^{2}}{B_{1} \Psi_{3}\left(3-u_{3}\right)(1+2 \rho)}\left[\frac{B_{2}}{B_{1}}+\frac{B_{1} u_{2}}{\left(2-u_{2}\right)}+1\right]
\end{aligned}
$$

where $\Psi_{\kappa}, \kappa \in\{2,3\}$, are given by (6)

Proof. The proof of the theorem follows from Lemma (4).

Theorem 8. If the function $f$ given by (1) belongs to the class $M_{\nu . q}^{\lambda}(\rho, t, \Psi)$, with $\Psi(\xi)$ satisfies Definition (1) and $\mu$, $B_{2} \in \mathbb{R}, 0 \leq \rho \leq 1$ and $|t| \leq 1$ but $t \neq 1$ then

(i) When $\sigma_{1}<\mu \leq \sigma_{3}$, we have

$$
\left|a_{3}-\mu a_{2}^{2}\right|+P\left[1-\frac{B_{2}}{B_{1}}-\frac{B_{1} u_{2}}{\left(2-u_{2}\right)}+\frac{\mu B_{1} \Psi_{3}\left(3-u_{3}\right)(1+2 \rho)}{\Psi_{2}^{2}(1+\rho)^{2}\left(2-u_{2}\right)^{2}}\right]\left|a_{2}^{2}\right| \leq Q
$$

(ii) When $\sigma_{3}<\mu \leq \sigma_{2}$, we have

$$
\left|a_{3}-\mu a_{2}^{2}\right|+P\left[1+\frac{B_{2}}{B_{1}}+\frac{B_{1} u_{2}}{\left(2-u_{2}\right)}-\frac{\mu B_{1} \Psi_{3}\left(3-u_{3}\right)(1+2 \rho)}{\Psi_{2}^{2}(1+\rho)^{2}\left(2-u_{2}\right)^{2}}\right]\left|a_{2}^{2}\right| \leq Q
$$

where

$$
\begin{gathered}
P=\frac{(1+\rho)^{2}\left(2-u_{2}\right)^{2} \Psi_{2}^{2}}{\Psi_{3} B_{1}(1+2 \rho)\left(3-u_{3}\right)}, \\
Q=\frac{B_{1}}{\Psi_{3}(1+2 \rho)\left(3-u_{3}\right)}
\end{gathered}
$$

where the values of $\sigma_{1}$ and $\sigma_{2}$ are defined by (19) and (20) accordingly.

$$
\sigma_{3}=\frac{\Psi_{2}^{2}(1+\rho)^{2}\left(2-u_{2}\right)^{2}}{B_{1} \Psi_{3}\left(3-u_{3}\right)(1+2 \rho)}\left[\frac{B_{2}}{B_{1}}+\frac{B_{1} u_{2}}{\left(2-u_{2}\right)}\right]
$$

and $\Psi_{\kappa}, \kappa \in\{2,3\}$ are given by (6).

Proof. Using Theorem 1, we can obtain (15) and (16) from (14) it is obvious that:

$$
C_{1}=\frac{2 a_{2}(1+\rho)\left(2-u_{2}\right) \Psi_{2}}{B_{1}}
$$

The proof follows from Lemma(5).

\section{Second Hankel Determinant}

Theorem 9. If $f(\xi) \in M_{\nu, q}^{\lambda}(\rho, t, \Psi)$ then

$$
\begin{aligned}
&\left|a_{2} a_{4}-a_{3}^{2}\right| \leq \mid \frac{B_{1}}{4}\left\{A\left[B_{1}\left(C_{3}+\frac{C_{1}^{3}}{4}-C_{1} C_{2}\right)+B_{2} C_{1}\left(C_{2}-\frac{C_{1}^{2}}{2}\right)\right]\right. \\
&+(E)\left[A\left(\left(2-u_{2}\right) u_{3}+(1-2 \rho)\left(3-u_{3}\right) u_{2}\right)\left(\frac{B_{1}^{2} C_{1}}{2(1+\rho)\left(3-u_{3}\right)\left(2-u_{2}\right)}\right)\right. \\
&\left.\left.-\frac{B_{1}}{(1+2 \rho)^{2}\left(3-u_{3}\right)^{2} \Psi_{3}^{2}}(E)\right]\right\} \mid
\end{aligned}
$$




$$
A=\frac{C_{1}}{(1+\rho)\left(2-u_{2}\right)(1+3 \rho)\left(4-u_{4}\right) \Psi_{2} \Psi_{4}}
$$

and

$$
E=C_{2}-\frac{C_{1}^{2}}{2}+\frac{1}{2} \frac{B_{2}}{B_{1}} C_{1}^{2}+\frac{B_{1} C_{1}^{2} u_{2}}{2\left(2-u_{2}\right)}
$$

Proof. From (14), (15) and (16), we have

$$
\begin{aligned}
\left|a_{2} a_{4}-a_{3}^{2}\right| \leq \mid \frac{B_{1}}{4}\left\{A\left[B_{1}\left(C_{3}+\frac{C_{1}^{3}}{4}-C_{1} C_{2}\right)+B_{2} C_{1}\left(C_{2}-\frac{C_{1}^{2}}{2}\right)\right]\right. & \\
+(E)\left[A\left(\left(2-u_{2}\right) u_{3}+(1-2 \rho)\left(3-u_{3}\right) u_{2}\right)\left(\frac{B_{1}^{2} C_{1}}{2(1+\rho)\left(3-u_{3}\right)\left(2-u_{2}\right)}\right)\right. & \left.\left.-\frac{B_{1}}{(1+2 \rho)^{2}\left(3-u_{3}\right)^{2} \Psi_{3}^{2}}(E)\right]\right\} \mid
\end{aligned}
$$

Where,

$$
A=\frac{C_{1}}{(1+\rho)\left(2-u_{2}\right)(1+3 \rho)\left(4-u_{4}\right) \Psi_{2} \Psi_{4}}
$$

and

Which gives the desired inequality (24)

$$
E=C_{2}-\frac{C_{1}^{2}}{2}+\frac{1}{2} \frac{B_{2}}{B_{1}} C_{1}^{2}+\frac{B_{1} C_{1}^{2} u_{2}}{2\left(2-u_{2}\right)}
$$

Corollary 10. If $\rho=0 ; t=0 ; u_{n}=1 ; C_{n}=\frac{(-1)^{n+1}}{(2 n) !}$ then

$$
\begin{aligned}
\left|a_{2} a_{4}-a_{3}^{2}\right| \leq & \mid \frac{B_{1}}{4}\left\{\frac{1}{6 \Psi_{2} \Psi_{4}}\left(\frac{77 B_{1}}{1440}-\frac{B_{2}}{12}\right)\right. \\
& +\left(\frac{-1}{6}+\frac{B_{2}}{8 B_{1}}+\frac{B_{1}}{8}\right)\left[\frac{B_{1}^{2}}{16 \Psi_{2} \Psi_{4}}-\frac{B_{1}}{4 \Psi_{3}^{2}}\left(\frac{-1}{6}+\frac{B_{2}}{8 B_{1}}\right.\right. \\
& \left.\left.\left.+\frac{B_{1}}{8}\right)\right]-\frac{B_{1}^{3}}{192 \Psi_{2} \Psi_{4}}\right\} \mid
\end{aligned}
$$

\section{Conclusion}

By setting the values of $\rho, t$ and $u_{n}$ we bring out the interesting coefficient inequality and subordination techniques, for the subclasses of $M_{\nu, q}^{\lambda}(\rho, t, \Psi)$. The investigation of initial coefficient bounds Fekete-Szego inequality and Second Hankel determinant for various subclasses can be a scope of future research for disorted signals.

\section{REFERENCES}

[1] Bulboaca T, "Differential subordinations and superordinations" in Recent Results, House of Scientific Book Publ. ClujNapoca, 2005.

[2] Miller S.S., Mocanu P.T, "Differential Subordinations: theory and applications" in Series on Monographs and Textbooks in Pure and Applied Mathematics, Vol. 225. Marcel Dekker Inc., New York and Basel, 2000.

[3] Szasz R., Kupan P.A, “About the univalence of the Bessel functions”, Studia Univ. Babes-Bolyai Math., vol. 54, no. 1, pp. 127-132, 2009.

[4] Baricz A, “Geometric properties of generalized Bessel functions”, Publ. Math. Debr., vol. 73, pp. 155-178, 2008.

[5] Jackson F.H, “The application of basic numbers to Bessel's and Legendre's functions”, Proc. Lond. Math. Soc., vol. 3, no. 2, pp. 1-23, 1905 .

[6] Selvakumaran K.A., Szász R, "Certain geometric properties of an integral operator involving Bessel functions”, Kyungpook Math. J., vol. 58, pp. 507-517, 2018.

[7] Carathéodory C, Über den Variabilitätsbereich der Koeffizienten von Potenzreihen, die gegebene Werte nicht annehmen”, Math. Ann., vol. 64, pp. 95-115, 1907. 
[8] Libera R.J., Zlotkiewicz E.J, "Coefficient bounds for the inverse of a function with derivative in P", Proc. Amer. Math. Soc., vol. 87, no. 2, pp. 251-257, 1983.

[9] Ma W., Minda D, "A unified treatment of some special classes of univalent functions", in Li, Z., Ren, F., Lang, L., Zhang, S. (eds.) Proceedings of the Conference on Complex Analysis, Tianjin, 1992, pp. 157-169. Conf. Proc. Lecture Notes Anal. I, Int. Press, Cambridge, 1994.

[10] Porwal S, "An application of a Poisson distribution series on certain analytic functions", J. Complex Anal., no. 1-3, 2014, Art. ID 984135. DOI: 10.1155/2014/984135

[11] Srivastava D., Porwal S, "Some sufficient conditions for Poisson distribution series associated with conic regions", Int. J. Adv. Technol. Eng. Sci., vol. 3, no. 1, pp. 229-235, 2015.

[12] Noonan J.W., Thomas D.K, "On the Second Hankel determinant of areally mean p-valent functions", Transactions of teh American Mathematical Society, vol. 223, pp. 337-346, 1976.

[13] GeorgiaIrina Oros, Alina Alb Lupas, "Sufficient conditions for univalence obtained by using Briot-Bouquet differential subordination", Mathematics and Statistics, vol. 8(2), pp. 126 - 136, 2020. DOI: 10.13189/ms.2020.080208

[14] Andy Liew Pik Hern, Aini Janteng, Rashidah Omar, "Hankel Determinant $H_{2}(3)$ for Certain Subclasses of Univalent Functions", Mathematics and Statistics vol. 8(5), pp. 566 - 569, 2020. DOI: 10.13189/ms.2020.080510 